\title{
Mechanisms of Tubulointerstitial Injury in the Kidney: Final Common Pathways to End-stage Renal Failure
}

\author{
Masaomi NANGAKU
}

\begin{abstract}
There are many different glomerular disorders, including glomerulonephritis, diabetic nephropathy, and hypertensive nephrosclerosis. However, once glomerular damage reaches a certain threshold, the progression of renal disease is consistent and irreversible. Recent studies emphasized the crucial role of tubulointerstitial injury as a mediator of progression of kidney disease. One common mechanism that leads to renal failure via tubulointerstitial injury is massive proteinuria. Accumulating evidence suggests critical effects of filtered macromolecules on tubular cells, including lysosomal rupture, energy depletion, and tubular injury directly induced by specific components such as complement components. Another common mechanism is chronic hypoxia in the tubulointerstitium. Tubulointerstitial damage results in the loss of peritubular capillaries, impairing blood flow delivery. Interstitial fibrosis also impairs oxygen diffusion and supply to tubular cells. This induces chronic hypoxia in this compartment, rendering a vicious cycle. Development of novel therapeutic approaches against these final common pathways will enable us to target any types of renal disease.
\end{abstract}

(Internal Medicine 43: 9-17, 2004)

Key words: proteinuria, hypoxia, complement, kidney failure

\section{Tubulointerstitial Damage Leading to Eventual Kidney Failure}

There are many different mechanisms that induce glomerular injury, including glomerulonephritis, hypertensive nephrosclerosis, and diabetic nephropathy. However, once renal damage reaches a certain threshold, progression of renal disease is consistent, irreversible, and largely independent of the initial insult (1).
Careful pathological analysis revealed that the impairment of renal function correlates better with the extent of tubulointerstitial damage than with the degree of glomerular damage (2-6). These pathological findings were supported by clinical observations that urinary markers of tubulointerstitial damage serve as reliable indicators of renal prognosis $(7,8)$.

Tubulointerstitial damage induces a decrease in GFR via several mechanisms. Tubular atrophy increases fluid delivery to the macula densa and triggers a reduction in GFR via tubuloglomerular feedback (9). Tubular damage also leads to atubular glomeruli and decreases the number of functional nephrons (10). In addition, it results in obliteration of postglomerular capillaries, leading to ischemic renal injury. Thus, it is widely recognized that tubulointerstitial injury serves as an important mediator and a final common pathway of chronic kidney failure (Fig. 1).

\section{One Final Common Pathway, Proteinuria- induced Tubular Damage}

One important mechanism that leads to eventual kidney failure via tubulointerstitial injury is massive proteinuria. While proteinuria had been considered merely a marker of glomerular damage, many studies indicate proteinuria as a cause of progression of renal injury (11).

A cohort of mass screening including a total of 107,192 subjects performed by Iseki and colleagues revealed that proteinuria was the most potent predictor of end-stage renal disease (ESRD) with an adjusted odds ratio of 14.9 (12). Large scale prospective studies including the MDRD study and the REIN study also established the relationship between proteinuria and progressive renal disease $(13,14)$. Systematic analyses of these studies revealed that greater urine protein excretion predicted a faster decline in $\operatorname{GFR}(15,16)$. Furthermore, recent analysis on the data of the REIN study revealed that short-term changes in proteinuria and the actual levels of residual proteinuria reliably predicted long-term disease progression, regardless of blood pressure control and treatment randomization (17). Multivariate analysis of the RENAAL study also demonstrated that proteinuria was an

Division of Nephrology and Endocrinology, University of Tokyo School of Medicine, Tokyo

Reprint requests should be addressed to Dr. Masaomi Nangaku, Division of Nephrology and Endocrinology, University of Tokyo School of Medicine, 7-3-1 Hongo, Bunkyo-ku, Tokyo 113-8655 


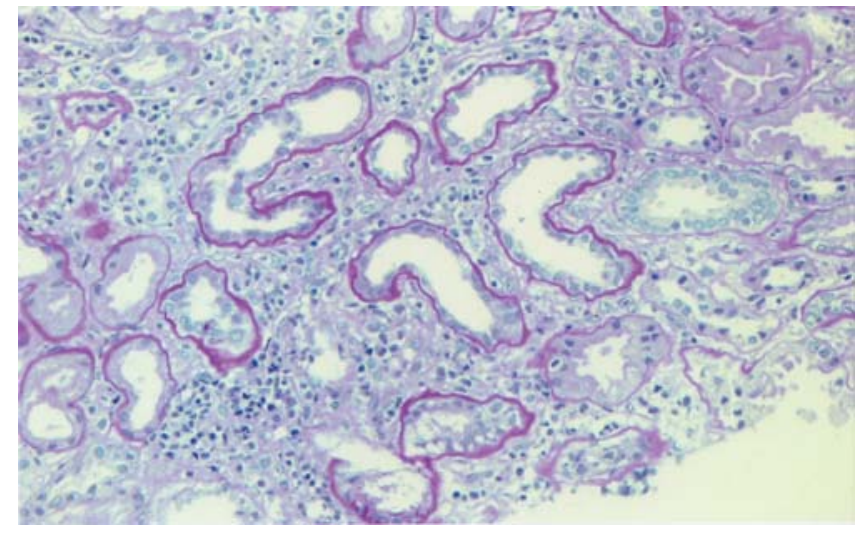

Figure 1. Tubulointerstitial injury in a 38-year-old male with chronic glomerulonephritis. In this PAS staining of a renal biopsy from a patient with IgA nephropathy and a serum creatinine level of $1.7 \mathrm{mg} / \mathrm{dl}$, note the prominent tubulointerstitial injury and fibrosis.

independent risk factor that predicts renal outcomes in type 2 diabetic patients with nephropathy (18).

Animal models with heavy proteinuria, a BSA overload model (19) and adriamycin nephrosis (20), show a large number of apoptotic cells in the tubulointerstitial compartment. Theoretically apoptosis of tubular cells leads to the formation of atrophic tubules and eventually generates atubular glomeruli. This speculation was confirmed by careful pathological analysis of animal models of progressive kidney failure with massive proteinuria, 5/6 nephrectomy (21) and uninephrectomized Heymann nephritis (22), showing that tubulointerstitial damage associated with proteinuria results in the formation of atubular glomeruli and interstitial fibrosis. Recent studies using stereologic methods on human biopsy samples revealed an increase in the number of atrophic tubules and the fractional volume of cortical interstitium in proteinuric diabetic nephropathy patients (23). A relationship between proteinuria and tubulointerstitial damage was also demonstrated in studies examining 78 biopsy samples of patients with membranous nephropathy (24).

More detailed analyses of animal models and human kidney biopsy samples revealed intracellular events induced by proteinuria. Proteinuria may contribute to tubulointerstitial damage by activation of transcriptional factors (AP-1 and NF-kB) (25) and upregulation of various proinflammatory and profibrotic genes (26). Inhibition of NF-kB improved tubulointerstitial injury in adriamycin nephrosis rats (27).

Proteinuria is also associated with the transdifferentiation of tubular cells into myofibroblasts. This phenotypic change is called "epithelial to mesenchymal transformation", and is believed to be a crucial step in fibrosis of the kidney. Two independent studies demonstrated transdifferentiation of tubular cells in the proteinuric phase of a remnant kidney model $(28,29)$.

All these studies showed that proteinuria activates and/or damages tubular and interstitial cells, leading to eventual kidney failure.

\section{What Kind of Proteinuria is Harmful?}

While patients with minimal change nephritic syndrome (MCNS) excrete a huge amount of protein in urine, they do not develop either tubulointerstitial damage or chronic renal failure. However, two characteristics of proteinuria in MCNS should be noted. Proteinuria of MCNS is highly selective, and patients with MCNS respond to steroid treatment, which means a relatively short duration of proteinuria in MCNS. Clinical data suggests that protracted and non-selective proteinuria induces tubulointerstitial injury and eventual kidney failure $(30,31)$. In patients with membranous nephropathy, IgA nephropathy, and focal segmental glomerulosclerosis, poor selectivity is significantly correlated with the severity of the tubulointerstitial damage and poor outcome (32-35).

\section{Mechanisms of Tubulointerstitial Damage by Proteinuria}

The next question is the mechanisms by which proteinuria results in tubular and interstitial damage. The mechanisms by which increased urinary protein concentrations lead to nephrotoxic injury are certain to be multifactorial and involve complex interactions between numerous pathways of cellular damage. Obstruction of tubular lumen by casts and obliteration of the tubular neck by glomerular tuft adhesions may contribute to tubulointerstitial damage by proteinuria. However, accumulating evidence emphasizes direct effects of filtered macromolecules on tubular cells (Fig. 2).

One hypothesis is that excess delivery of protein damages tubular cells in a non-specific manner and leads to tubulointerstitial injury (36-38). Reabsorption of excessive quantities of protein overloads the lysosomal pathways in the tubular cells, leading to eventual lysosomal rupture. This idea is appealing, but lacks experimental support. Excessive metabolic demands result in energy depletion. This hypothesis is also controversial because the metabolic work of protein reabsorption can be relatively modest compared with the amount of energy required to absorb other substrates such as sodium, glucose, and bicarbonate (39).

Various components in the proteinuric urine may damage tubular cells directly. These include growth factors, transferrin, albumin, albumin-bound fatty acids, and complement components. Toxic effects of these specific components are the focus of current investigation. Complement components in the proteinuric urine are believed to play an especially crucial role, and this topic is discussed in a separate section.

Albumin is one of the major components in proteinuric urine. Albumin stimulates cultured proximal tubules to express cytokines and chemokines via reactive oxygen species generation $(40,41)$. Functional significance of proteinuriainduced chemokine was emphasized by Shimizu from 


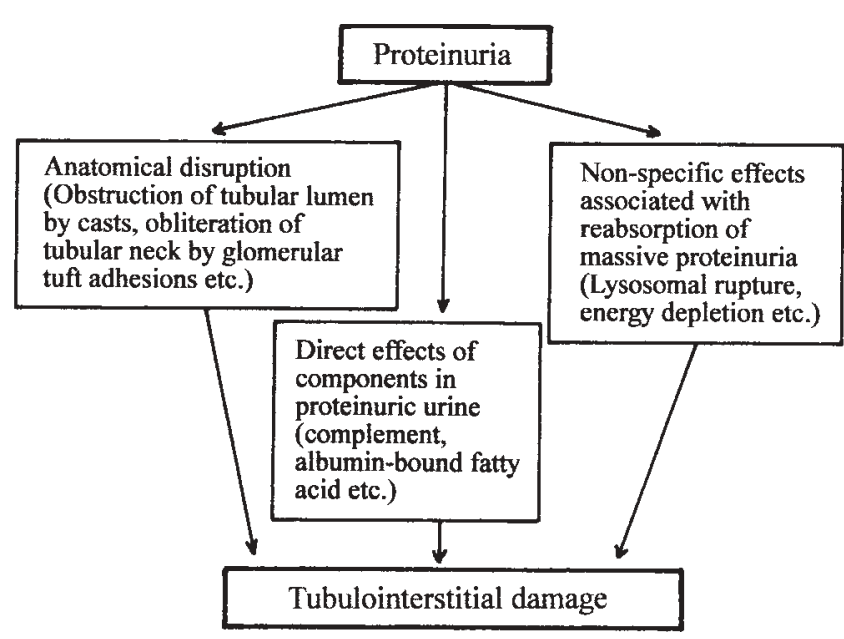

Figure 2. Schematic view of mechanisms of proteinuriainduced tubulointerstitial injury. Although proteinuria damages tubular cells via various mechanisms, accumulating evidence emphasizes crucial roles of direct effects of components in proteinuric urine, such as complement components.

Matsuo's group, who showed that blockade of MCP-1 in a proteinuric BSA overload model attenuated tubulointerstitial injury (42). However, recent studies emphasized a role of albumin-bound fatty acids rather than albumin itself in mediating tubulointerstitial injury. Kamijo from Kimura's group demonstrated an important role of albumin-bound fatty acids in her studies employing a BSA overload model with fatty acid depleted and fatty acid repleted albumin (43). Arici from Brunskill's group showed that albumin-bound fatty acids stimulate PPAR and subsequently induce apoptosis in a dose-dependent manner in cultured proximal tubular cells (44).

\section{Activation of Complement Components in Proteinuric Urine}

Protein overload in tubular cells is associated with ammonium production. Complement component C3 modified by ammonia is called amidated $\mathrm{C} 3$, and amidated $\mathrm{C} 3$ forms the alternative pathway convertase of the complement cascade. Preferential secretion of ammonia into the tubular lumen leads to inappropriate activation of the alternative pathway at the brush border in proteinuric conditions.

Studies utilizing rats with remnant kidneys localized C3 staining in the brush border or within the cytoplasm at sites of high protein reabsorption, and C3 deposition in this model was ameliorated by reduction of proteinuria with ACE inhibitor $(45,46)$. Matsuo's group performed animal experiments to clarify functional roles of complement components in proteinuric urine $(47,48)$. In proteinuric animals, pharmacological complement depletion improved tubulointerstitial injury. Matsuo's group also demonstrated complement activation products in urine of patients with various glomerular diseases (49). The degree of intratubular complement activation correlated with the level of non-selective proteinuria.

We also demonstrated a crucial role of complement components, especially C5b-9, in proteinuric urine in tubulointerstitial injury induced by proteinuria. To elucidate roles of complement in proteinuric urine, we induced puromycin nephrosis and a remnant kidney model in genetically C6 deficient rats. While $\mathrm{C} 6$ deficient rats can produce inflammatory mediators such as $\mathrm{C} 3 \mathrm{a}$ and $\mathrm{C} 5 \mathrm{a}$, they can not generate C5b-9, the terminal complex of the complement cascade. We observed that massive proteinuria induced tubulointerstitial injury associated with a marked deposition of C5b-9 on the apical membrane of proximal tubular cells in complement sufficient rats. In contrast, the tubulointerstitial damage was less severe in rats without C5b-9 formation $(50,51)$.

Hosts are endowed with endogenous complement regulatory proteins to protect themselves against inappropriate complement activation (52). We also demonstrated protective effects of an endogenous complement regulatory protein (Crry) in tubules against the harmful effects of tubular complement activation in the setting of massive proteinuria utilizing in vivo antisense approaches (53).

Thus, intraluminal activation of the terminal complement cascade leading to the formation of the C5b-9 membrane attack complex is a crucial mediator of tubulointerstitial damage and progressive renal failure in proteinuric renal diseases irrespective of the type of primary glomerular injury.

\section{Another Final Common Pathway, Chronic Hypoxia}

In some human diseases such as hypertensive nephrosclerosis, we rarely see massive proteinuria. But the patients develop tubulointerstitial damage and eventual kidney failure. This can be explained by another common pathway, chronic ischemic damage in the tubulointerstitium (54-56).

Despite a high overall oxygen supply, the tissue oxygen tension in the kidney is comparatively low due to shunt diffusion of oxygen between arterial and venous vessels that run in parallel in close contact. Oxygen tension in the renal medulla is continuously below $10 \mathrm{mmHg}$, while oxygen tension in the renal cortex is more variable, with an average $\mathrm{PO}_{2}$ of around $30 \mathrm{mmHg}$. However, the oxygen concentration in the cortex decreases dramatically in accordance with changes in renal perfusion while the oxygen concentration in the medulla is relatively preserved (57). Thus, the kidney is rather sensitive to changes in oxygen delivery. While this sensitivity is reflected by the ability of the kidney to adjust the production of EPO to changes in oxygen supply, it also renders the kidney prone to hypoxic injury.

Chronic ischemia in the tubulointerstitium can occur via several mechanisms such as intrarenal vasoconstriction (secondary to local activation of renin-angiotensin system or loss of vasodilatory nitric oxide) or structural lesions that impair blood flow delivery (Fig. 3). Impairment of the glomerular capillary bed, as it occurs in glomerulosclerosis, auto- 


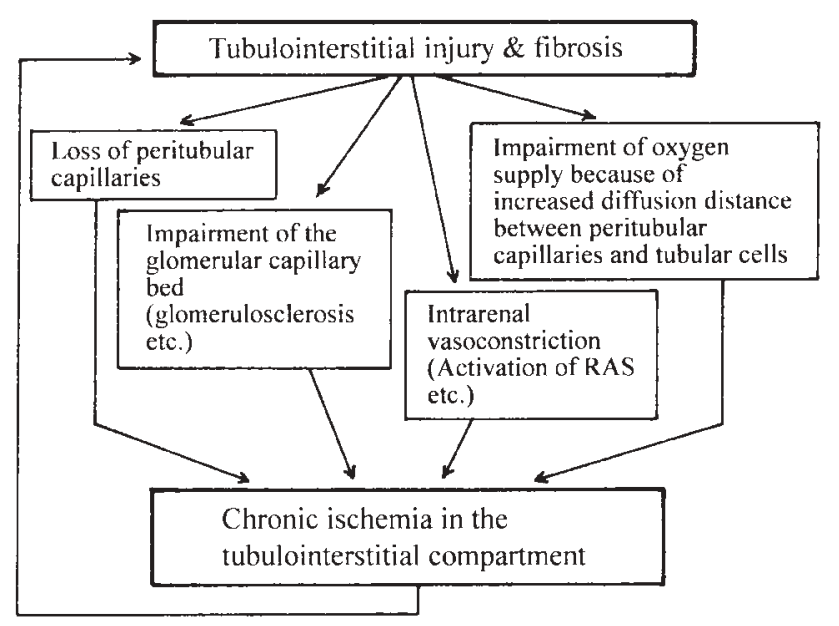

Figure 3. Schematic view of mechanisms of chronic hypoxiainduced tubulointerstitial injury. Chronic hypoxia and tubulointerstitial injury composes a vicious cycle, leading to eventual end-stage kidney failure.

matically impairs peritubular perfusion and thus tubular oxygen supply. Tubulointerstitial damage also results in the loss of peritubular capillaries in association with a decrease in blood flow delivery to the corresponding region. Furthermore, interstitial fibrosis impairs tubular oxygen supply because the size of the interstitial compartment determines diffusion distance between peritubular capillaries and tubular cells. These mechanisms induce chronic hypoxia in this compartment, rendering a vicious cycle.

\section{Loss of Peritubular Capillaries in Chronic Renal Disease}

Histological studies of human kidneys confirmed that peritubular capillary loss is correlated with interstitial fibrosis and tubular atrophy $(58,59)$. The significance of peritubular capillary damage, with subsequent tissue hypoxia and ischemia, has been emphasized by recent findings in animal models. Ohashi et al suggested an important role of peritubular capillary disruption in the development of renal disease and in the impairment of renal function in an antiGBM nephritis model in Wistar Kyoto rats (60). Statistical analysis of this model revealed significant correlation between the peritubular capillary number and indicators of renal function.

We performed blockade of nitric oxide as a potent vasodilator and platelet inhibitory factor in our thrombotic microangiopathy model, and demonstrated that inhibition of eNOS markedly exacerbated the tubulointerstitial injury (61). We also studied genetically engineered rats with or without expression of endothelin receptor type B (62). This receptor is known to mediate vasodilatory and proliferative effects of endothelin. When we induced thrombotic microangiopathy in these rats, rats without endothelin receptor type B developed more severe kidney damage with loss of peritubular capillaries and deterioration of renal function.

The remnant kidney model was associated with progressive capillary loss and renal scarring. Kang from Johnson's group showed that the loss of capillaries is correlated with a loss of VEGF in the kidney, conditions that favor endothelial cell loss and impaired angiogenesis (63). A reduction in the peritubular capillary density was also observed after severe ischemic insult, resulting in a persistent reduction in $\mathrm{PO}_{2}$ and progression of renal failure $(64,65)$.

All these experimental data suggest that the maintenance of the microvasculature is critical for the prevention of progression of kidney disease.

\section{Tubulointerstitial Hypoxia in Chronic Renal Disease}

In pigs with hypercholesterolemic diets and/or renal artery stenosis, low cortical perfusion demonstrated by electronbeam computed tomography was associated with renal fibrosis, tissue oxidative stress, and reduction in GFR (66). Matsumoto from our group showed hypoxia in the tubulointerstitial compartment in a model of chronic progressive glomerulonephritis in rats (Matsumoto M, Nangaku M. manuscript in preparation). Furthermore, we also demonstrated that hypoxia in the tubulointerstitium precedes histological damage in the corresponding compartment in a chronic kidney failure model (Manotham K, Nangaku M. manuscript in preparation).

Considering the role of ischemia in tubulointerstitial injury, one may wonder whether anemia in kidney disease can accelerate the decline in renal function. The retrospective multivariate logistic analyses of 71,802 subjects in Japan showed that anemia was an independent risk factor of endstage renal disease (ESRD) (67). Anemia also turned out to be an independent risk factor that significantly predicted doubling of serum creatinine or ESRD in patients with type 2 diabetes in whom blood pressure was controlled (18).

\section{Mechanisms of Tubulointerstitial Injury by Hypoxia}

Mechanisms of tubulointerstitial damage induced by hypoxia are multifactorial. Hypoxia can activate fibroblasts, change extracellular matrix metabolism of resident renal cells, and lead to eventual fibrogenesis (68-70). Furthermore, Manotham from our group showed that mild hypoxia can induce transdifferentiation of cultured tubular cells into myofibroblasts (71). Transdifferentiation of tubular cells into myofibroblasts in a remnant kidney model was demonstrated as described above $(28,29)$, and it was proposed that proteinuria might play a role in this transformation. However, the phenotypic transformation in this model occurred in a relative late stage, suggesting a possible involvement of chronic hypoxia in addition to proteinuria in transdifferentia- 
tion of tubular cells in vivo. A vicious cycle exists with hypoxia promoting interstitial fibrosis and increased matrix deposition, in turn further impairing peritubular blood flow and oxygen supply.

Renal tubular cells subjected to hypoxia have profound functional deficits of their mitochondria and persistent energy deficits (72). Tanaka from our group recently demonstrated that hypoxia induces apoptosis of renal tubular and endothelial cells via the mitochondrial pathways $(73,74)$. Histological analysis of a murine model confirmed that hypoxia induces apoptosis of renal tubular cells with tubular atrophy in vivo (75).

These studies clarified the crucial roles of chronic ischemia due to derangement of capillaries as a mediator of progression of end-stage renal disease.

\section{Treatment Targeting Proteinuric Tubulointerstitial Damage}

Understanding of mechanisms of tubulointerstitial injury helps us to develop novel therapeutic approaches against chronic renal failure. ACEIs and ARBs are now the golden standard of therapies against proteinuric renal disease. Renoprotective effects of these reagents had been attributed to amelioration of intraglomerular hypertension. However, recent studies demonstrated that inhibition of RAS improves the molecular mechanisms to retain glomerular permeability and reduces the amount of proteinuria. ACEIs and ARBs induce redistribution of the molecules in the slit diaphragm $(76,77)$, and improve selectivity of proteinuria in patients with glomerular diseases $(78,79)$. Large scale clinical trials and a meta analysis confirmed the benefits of ACEIs against a variety of renal diseases with their antiproteinuric effects $(14,80,81)$. Furthermore, recent studies demonstrated that the greater beneficial effect of ACEIs in renal disease patients with higher baseline proteinuria could be explained by the greater antiproteinuric effects in these patients (82). Thus, one of the protective mechanisms of ACEIs and ARBs is the reduction of the amount of proteinuria.

However, these drugs do not completely inhibit the progression of chronic renal disease. Therapeutic approaches against specific components in proteinuric urine may have additive beneficial effects. As amelioration of inappropriate complement activation retards progression of chronic proteinuric renal disease in animals, the potential of using complement regulators to modify renal disease exists not just in immunological kidney disease but possibly also in chronic non-immunological proteinuric renal injury (83).

\section{Treatment Targeting Hypoxic Tubulointerstitial Damage}

As anemia is a risk factor for renal failure, correction of anemia by EPO and the subsequent improvement in oxygenation of the kidney should theoretically retard progression of renal failure. One retrospective study (84) and two prospective studies $(85,86)$ suggested that improvement of anemia by treatment with EPO delayed the progression of renal failure.

While an increase in the number of erythrocytes by EPO improves oxygen delivery to organs, cells can adjust to hypoxic conditions by various mechanisms.

VEGF treatment reduces fibrosis and stabilizes renal function in the remnant kidney model (87). Administration of VEGF also restores the number of peritubular capillaries and protects the kidney in the thrombotic microangiopathy model $(88,89)$. These beneficial effects of VEGF were probably mediated by preservation of the capillary endothelium and were associated with partial reversal of the impaired angiogenesis. However, because the formation of a functionally intact microvasculature requires coordinated activation of several genes, vessels induced by overexpression of a single gene such as VEGF may be leaky, immature, or irregular. Recent studies by Floege's group demonstrated aggravation of mesangial injury by treatment with VEGF in anti-Thy1 nephritis, cautioning against therapeutic approaches utilizing VEGF (90). It is desirable to activate a "master gene" switch that results in a broad and coordinated downstream reaction to protect tissues against hypoxia.

One of the most important factors in the cellular response to hypoxia is hypoxia-inducible factor, HIF (91). HIF binds to the hypoxia responsive element in the cis-regulatory regions of its target genes, and transcriptionally activates genes encoding proteins that mediate adaptive responses to reduced oxygen availability. Under normoxic conditions, the binding of pVHL to HIF1-alpha, which is hydroxylated on conserved prolyl residues, directs the polyubiquitination and proteasomal degradation of the latter. Under hypoxic conditions, the prolyl hydroxylase enzyme cannot hydroxylate HIF, and therefore HIF is not recognized by pVHL. As a result, HIF accumulates in the cell and is available to activate transcription. Therefore, stimulation of HIF-1 signaling can be theoretically more effective in ischemic states because it can induce expression of a variety of oxygen-regulated and renoprotective genes.

Prolyl hydroxylase inhibitors are the focus of recent studies as a novel strategy to stabilize HIF, and a variety of new compounds are now being developed (92). Local injection of these inhibitors increased invasion of highly vascularized tissue in a sponge model of angiogenesis (93). Polypeptides containing the HIF prolyl hydroxylation motifs fused to a nuclear translocation signal were shown to compete with endogenous HIF for its degradative pathway, resulting in HIF stabilization and induction of angiogensis in vivo (94). In models such as hindlimb ischemia, injection of DNA expressing a constitutively active fusion protein containing the $\mathrm{N}$-terminal half of HIF-1alpha and VP16 was more effective than a VEGF-expressing vector in restoring blood flow (9597).

The prolyl hydroxylation requires iron as a cofactor, and this requirement explains the hypoxia-mimetic effects of iron chelators such as deferoxamine and iron antagonists such as 

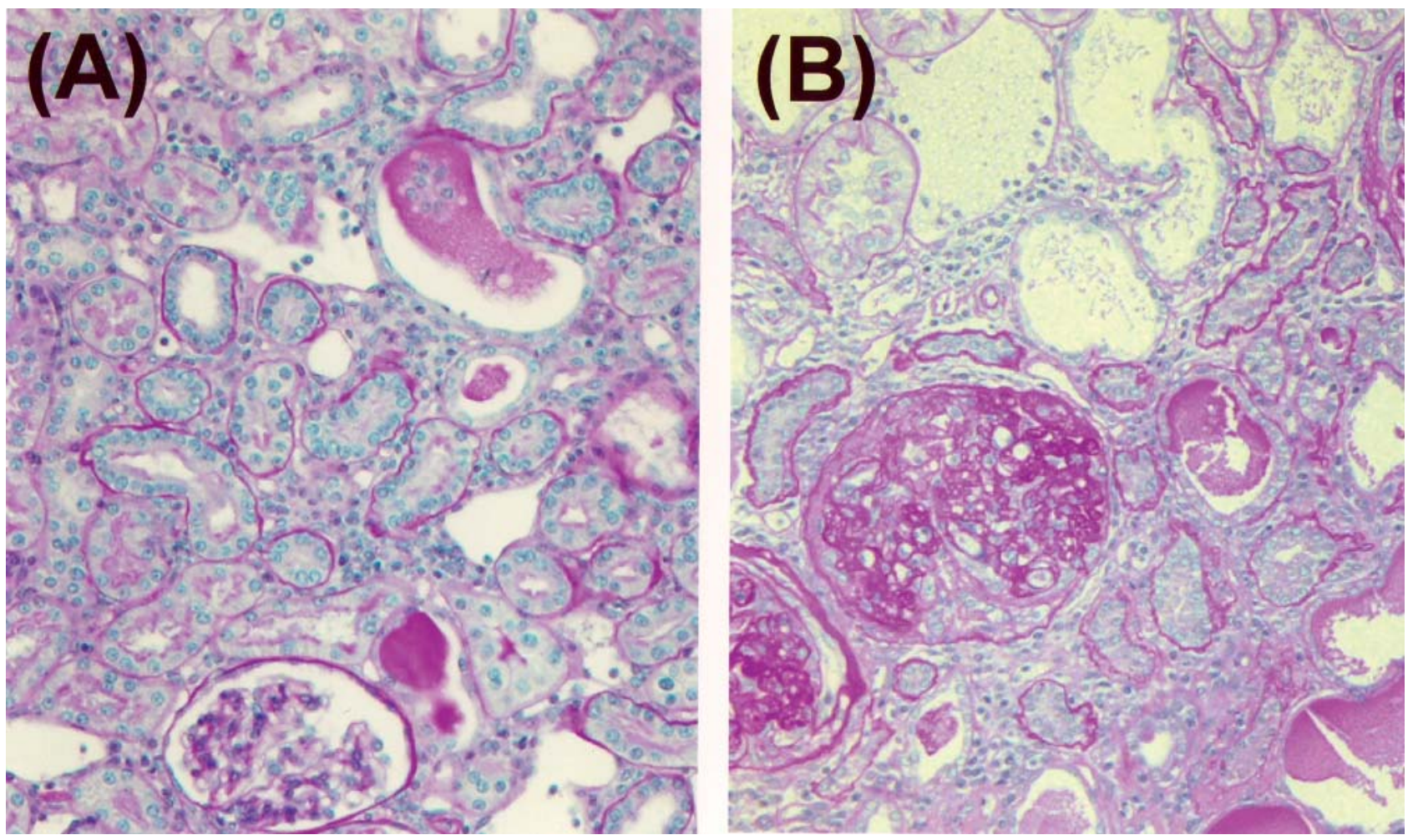

Figure 4. Tubulointerstitial injury in animal models. (A) Puromycin nephrosis model (3 weeks): This is a good model of proteinuria-induced tubulointerstitial injury. Morphology of the glomerulus remains relatively intact. (B) Remnant kidney model (4 months): Tubulointerstitial injury in this model is associated with proteinuria, hypertension, glomerulosclerosis, and loss of peritubular capillaries.

cobalt chloride. Cobalt chloride activates the HIF pathway not only by antagonizing this reaction but also by inhibition of the interaction between HIF-alpha and von Hippel-Lindau protein (98). We recently demonstrated renoprotective effects of stimulation of HIF-1 signaling utilizing cobaltous chloride administration in an ischemic model of renal injury (99). Administration of cobalt induced up-regulation of HIFregulated genes, such as VEGF and EPO, and subsequently improved the tubulointerstitial damage induced by hypoxia.

\section{Summary and Conclusion}

Tubulointerstitial injury is a final common pathway leading to end-stage renal failure. This is mediated by massive proteinuria containing a large amount of complement components and chronic hypoxia with loss of peritubular capillaries in the tubulointerstitium. Relative contributions of these pathways may vary among different clinical entities and individual cases (Fig. 4). Further studies are awaited to develop powerful therapeutic approaches against these final common pathways, which can target any types of renal disease.
Acknowledgments: The author is very grateful to Dr. William G. Couser (University of Washington, Seattle, WA), Dr. Kiyoshi Kurokawa (Tokai University, Isehara, Japan), and Dr. Toshiro Fujita (University of Tokyo, Tokyo, Japan) for their generous support. The author is also grateful to Drs. Richard J. Johnson (University of Florida, Gainesville, FL), Reiko Inagi (Tokai University, Isehara, Japan), Toshio Miyata (University of Tokai, Isehara, Japan), and Stuart J. Shankland (University of Washington, Seattle, WA) for their continuous support. Particular thanks are due to my friends and colleagues: Takamoto Ohse, Tetsuhiro Tanaka, Ichiro Kojima, Makiko Matsumoto, Jing Shao, Krissanapong Manotham. The author would like to acknowledge research grants from the Japanese Ministry of Health, Labour and Welfare and NOVARTIS Foundation (Japan) for the Promotion of Science.

\section{References}

1) Nangaku M. Final common pathways of progression of renal diseases. Clin Exp Nephrol 6: 182-189, 2002.

2) Nath KA. Tubulointerstitial changes as a major determinant in the progression of renal damage. Am J Kidney Dis 20: 1-17, 1992.

3) Risdon RA, Sloper JC, de Wardener HE. Relationship between renal function and histological changes found in renal biopsy specimens from patients with persistent glomerular nephritis. Lancet 2: 363-366, 1968.

4) Schainuck LI, Striker GE, Cutler RE, Benditt EP. Structural-functional correlations in renal disease. II. The correlations. Human Pathol 1: 631-641, 1970.

5) Striker GE, Schainuck LI, Cutler RE, Benditt EP. Structural-functional 


\section{Tubulointerstitial Injury and ESRD}

correlations in renal disease. I. A method for assaying and classifying histopathologic changes in renal disease. Human Pathol 1: 615-630, 1970.

6) Mackensen-Haen S, Bader R, Grund KE, Bohle A. Correlations between renal cortical interstitial fibrosis, atrophy of proximal tubules and impairment of glomerular filtration rate. Clin Nephrol 15: 167-171, 1981.

7) Woo KT, Lau YK, Lee GS, Wei SS, Lim CH. Pattern of proteinuria in IgA nephritis by SDS-PAGE: clinical significance. Clin Nephrol 36: 6-11, 1991.

8) Bazzi C, Petrini C, Rizza V, Arrigo G, Beltrame A, D’Amico G. Characterization of proteinuria in primary glomerulonephritides. SDSPAGE patterns: clinical significance and prognostic value of low molecular weight (“tubular") proteins. Am J Kidney Dis 29: 27-35, 1997.

9) Bohle A, Backensen-Haen S, von Gise H. Significance of tubulointerstitial changes in the renal cortex for the excretory function and concentration ability of the kidney. Am J Nephrol 7: 421-433, 1987.

10) Marcussen N. Atubular glomeruli and the structural basis for chronic renal failure. Lab Invest 66: 265-284, 1992.

11) Remuzzi G, Bertani T. Pathophysiology of progressive nephropathies. N Engl J Med 339: 1448-1456, 1998.

12) Iseki K, Iseki $C$, Ikemiya $Y$, Fukiyama K. Risk of developing end-stage renal disease in a cohort of mass screening. Kidney Int 49: 800-805, 1996.

13) Klahr S, Levey AS, Beck GJ, et al. The effects of dietary protein restriction and blood-pressure control on the progression of chronic renal disease. Modification of diet in Renal Disease Study Group. N Engl J Med 330: 877-884, 1994.

14) Ruggenenti P, Perna A, Gherardi G, Gaspari F, Benini R, Remuzzi G. Renal function and requirement for dialysis in chronic nephropathy patients on long-term ramipril: REIN follow-up trial. Gruppo Italiano di Studi Epidemiologici in Nefrologia (GISEN). Ramipril efficacy in nephropathy. Lancet 352: 1252-1256, 1998.

15) Hunsicker LG, Adler S, Caggiula A, et al. Predictors of the progression of renal disease in the modification of diet in Renal Disease Study. Kidney Int 51: 1908-1919, 1997.

16) Ruggenenti P, Perna A, Mosconi L, Pisoni R, Remuzzi G. Urinary protein excretion rate is the best independent predictor of ESRF in nondiabetic proteinuric chronic nephropathies. "Gruppo Italiano di Studi Epidemiologici in Nefrologia" (GISEN). Kidney Int 53: 1209-1216, 1998.

17) Ruggenenti P, Perna A, Remuzzi G. Retarding progression of chronic renal disease: The neglected issue of residual proteinuria. Kidney Int 63: 2254-2261, 2003.

18) Keane WF, Brenner BM, de Zeeuw D, et al, RENAAL Study Investigators. The risk of developing end-stage renal disease in patients with type 2 diabetes and nephropathy: the RENAAL study. Kidney Int $\mathbf{6 3}$ : 1499-1507, 2003.

19) Thomas ME, Brunskill NJ, Harris KP, et al. Proteinuria induces tubular cell turnover: A potential mechanism for tubular atrophy. Kidney Int 55: 890-898, 1999.

20) Shu Y, Hoshi S, Tomari S, Watanabe T, Nagata M. Phenotypic changes and cell cycle activation in early tubulointerstitial injury of rat adriamycin nephrosis. Pathol Int 52: 214-223, 2002.

21) Gandhi M, Olson JL, Meyer TW. Contribution of tubular injury to loss of remnant kidney function. Kidney Int 54: 1157-1165, 1998.

22) Benigni A, Gagliardini E, Remuzzi A, Corna D, Remuzzi G. Angiotensin-converting enzyme inhibition prevents glomerular-tubule disconnection and atrophy in passive Heymann nephritis, an effect not observed with a calcium antagonist. Am J Pathol 159: 1743-1750, 2001.

23) Najafian B, Kim Y, Crosson JT, Mauer M. Atubular glomeruli and glomerulotubular junction abnormalities in diabetic nephropathy. J Am Soc Nephrol 14: 908-917, 2003.

24) Magil AB. Tubulointerstitial lesions in human membranous glomerulonephritis: relationship to proteinuria. Am J Kidney Dis 25: 375-379, 1995.
25) Mezzano SA, Barria M, Droguett MA, et al. Tubular NF-kappaB and AP-1 activation in human proteinuric renal disease. Kidney Int $\mathbf{6 0}$ : 1366-1377, 2001.

26) Eddy AA, Giachelli CM. Renal expression of genes that promote interstitial inflammation and fibrosis in rats with protein-overload proteinuria. Kidney Int 47: 1546-1557, 1995.

27) Rangan GK, Wang Y, Tay YC, Harris DC. Inhibition of nuclear factorkappaB activation reduces cortical tubulointerstitial injury in proteinuric rats. Kidney Int 56: 118-134, 1999.

28) Ng YY, Huang TP, Yang WC, et al. Tubular epithelial-myofibroblast transdifferentiation in progressive tubulointerstitial fibrosis in $5 / 6$ nephrectomized rats. Kidney Int 54: 864-876, 1998.

29) Abbate M, Zoja C, Rottoli D, Corna D, Tomasoni S, Remuzzi G. Proximal tubular cells promote fibrogenesis by TGF-beta1-mediated induction of peritubular myofibroblasts. Kidney Int 61: 2066-2077, 2002.

30) D’Amico G, Bazzi C. Pathophysiology of proteinuria. Kidney Int 63: 809-825, 2003.

31) Tencer J, Bakoush O, Torffvit O. Diagnostic and prognostic significance of proteinuria selectivity index in glomerular diseases. Clin Chim Acta 297: 73-83, 2000.

32) Bakoush O, Torffvit O, Rippe B, Tencer J. High proteinuria selectivity index based upon IgM is a strong predictor of poor renal survival in glomerular diseases. Nephrol Dial Transplant 16: 1357-1363, 2001.

33) Bazzi C, Petrini C, Rizza V, Arrigo G, D’Amico G. A modern approach to selectivity of proteinuria and tubulointerstitial damage in nephrotic syndrome. Kidney Int 58: 1732-1741, 2000.

34) Bazzi C, Petrini C, Rizza V, et al. Urinary excretion of IgG and alpha(1)-microglobulin predicts clinical course better than extent of proteinuria in membranous nephropathy. Am J Kidney Dis 38: 240248, 2001.

35) Bazzi C, Petrini C, Rizza V, et al. Fractional excretion of IgG predicts renal outcome and response to therapy in primary focal segmental glomerulosclerosis: a pilot study. Am J Kidney Dis 41: 328-335, 2003.

36) Ruggenenti P, Remuzzi G. The role of protein traffic in the progression of renal diseases. Annu Rev Med 51: 315-327, 2000.

37) Remuzzi G, Ruggenenti P, Perico N. Chronic renal diseases: renoprotective benefits of renin-angiotensin system inhibition. Ann Intern Med 136: 604-615, 2002.

38) Brunskill NJ. Mechanisms of albumin uptake by proximal tubular cells. Am J Kidney Dis 37 (1 Suppl 2): S17-S20, 2001.

39) Meyer TW. Tubular injury in glomerular disease. Kidney Int 63: 774 787, 2003.

40) Wang Y, Rangan GK, Tay YC, Wang Y, Harris DC. Induction of monocyte chemoattractant protein-1 by albumin is mediated by nuclear factor kappaB in proximal tubule cells. J Am Soc Nephrol 10: 1204 1213, 1999.

41) Tang S, Leung JC, Abe K, et al. Albumin stimulates interleukin-8 expression in proximal tubular epithelial cells in vitro and in vivo. $\mathrm{J}$ Clin Invest 111: 515-527, 2003.

42) Shimizu H, Maruyama S, Yuzawa $Y$, et al. Anti-monocyte chemoattractant protein-1 gene therapy attenuates renal injury induced by protein-overload proteinuria. J Am Soc Nephrol 14: 1496-1505, 2003.

43) Kamijo A, Kimura K, Sugaya T, et al. Urinary free fatty acids bound to albumin aggravate tubulointerstitial damage. Kidney Int 62: 16281637, 2002.

44) Arici M, Chana R, Lewington A, Brown J, Brunskill NJ. Stimulation of proximal tubular cell apoptosis by albumin-bound fatty acids mediated by peroxisome proliferator activated receptor-gamma. J Am Soc Nephrol 14: 17-27, 2003.

45) Abbate M, Zoja C, Rottoli D, et al. Antiproteinuric therapy while preventing the abnormal protein traffic in proximal tubule abrogates protein- and complement-dependent interstitial inflammation in experimental renal disease. J Am Soc Nephrol 10: 804-813, 1999.

46) Abbate M, Zoja C, Corna D, Capitanio M, Bertani T, Remuzzi G. In progressive nephropathies, overload of tubular cells with filtered 
proteins translates glomerular permeability dysfunction into cellular signals of interstitial inflammation. J Am Soc Nephrol 9: 1213-1224, 1998.

47) Nomura A, Morita Y, Maruyama S, et al. Role of complement in acute tubulointerstitial injury of rats with aminonucleoside nephrosis. Am J Pathol 151: 539-547, 1997.

48) Morita Y, Nomura A, Yuzawa Y, et al. The role of complement in the pathogenesis of tubulointerstitial lesions in rat mesangial proliferative glomerulonephritis. J Am Soc Nephrol 8: 1363-1372, 1997.

49) Morita Y, Ikeguchi H, Nakamura J, Hotta N, Yuzawa Y, Matsuo S. Complement activation products in the urine from proteinuric patients. J Am Soc Nephrol 11: 700-707, 2000.

50) Nangaku M, Pippin J, Couser WG. C6 mediates chronic progression of tubulointerstitial damage in rats with remnant kidneys. J Am Soc Nephrol 13: 928-936, 2002.

51) Nangaku M, Pippin J, Couser WG. Complement membrane attack complex (C5b-9) mediates interstitial disease in experimental nephrotic syndrome. J Am Soc Nephrol 10: 2323-2331, 1999.

52) Nangaku M. Complement regulatory proteins in glomerular diseases. Kidney Int 54: 1419-1428, 1998.

53) Hori Y, Yamada K, Hanafusa N, et al. Crry, a complement regulatory protein, modulates renal interstitial disease induced by proteinuiria. Kidney Int 56: 2096-2106, 1999.

54) Kang DH, Kanellis J, Hugo C, et al. Role of the microvascular endothelium in progressive renal disease. J Am Soc Nephrol 13: 806-816, 2002.

55) Fine LG, Bandyopadhay D, Norman JT. Is there a common mechanism for the progression of different types of renal diseases other than proteinuria? Towards the unifying theme of chronic hypoxia. Kidney Int 75: S22-S26, 2000.

56) Eckardt KU, Rosenberger C, Jurgensen JS, Wiesener MS. Role of hypoxia in the pathogenesis of renal disease. Blood Purif 21: 253-257, 2003.

57) Dobrowolski L, Flemming B, Wronski T. Tissue $\mathrm{pO}_{2}$ in the renal cortex and medulla of anaesthetised rats: effect of fluctuations in renal perfusion pressure. Nephrol Dial Transplant 18 (Suppl 4): 9, 2003 (Abstract).

58) Bohle A, Mackensen-Haen S, Wehrmann M. Significance of postglomerular capillaries in the pathogenesis of chronic renal failure. Kidney Blood Press Res 19: 191-195, 1996.

59) Choi YJ, Chakraborty S, Nguyen V, et al. Peritubular capillary loss is associated with chronic tubulointerstitial injury in human kidney: Altered expression of vascular endothelial growth factor. Human Pathol 31: 1491-1497, 2000.

60) Ohashi R, Kitamura H, Yamanaka N. Peritubular capillary injury during the progression of experimental glomerulonephritis in rats. J Am Soc Nephrol 11: 47-56, 2000.

61) Shao J, Miyata T, Yamada K, et al. A protective role of nitric oxide in a model of thrombotic microangiopathy in rats. J Am Soc Nephrol 12 : 2088-2097, 2001.

62) Nangaku M, Yamada K, Gariepy CE, et al. ETB receptor protects the tubulointerstitium in experimental thrombotic microangiopathy. Kidney Int 62: 922-928, 2002.

63) Kang DH, Joly AH, Oh SW, et al. Impaired angiogenesis in the remnant kidney model: I. Potential role of vascular endothelial growth factor and thrombospondin-1. J Am Soc Nephrol 12: 1434-1447, 2001.

64) Basile DP, Donohoe D, Roethe K, Osborn JL. Renal ischemic injury results in permanent damage to peritubular capillaries and influences long-term function. Am J Physiol Renal Physiol 281: F887-F899, 2001.

65) Basile DP, Donohoe DL, Roethe K, Mattson DL. Chronic renal hypoxia after acute ischemic injury: effects of L-arginine on hypoxia and secondary damage. Am J Physiol Renal Physiol 284: F338-F348, 2003.

66) Chade AR, Rodriguez-Porcel M, Grande JP, et al. Distinct renal injury in early atherosclerosis and renovascular disease. Circulation 106: $1165-1171,2002$
67) Iseki K, Ikemiya Y, Iseki C, Takishita S. Haematocrit and the risk of developing end-stage renal disease. Nephrol Dial Transplant 18: 899905, 2003.

68) Norman JT, Orphanides C, Garcia P, Fine LG. Hypoxia-induced changes in extracellular matrix metabolism in renal cells. Exp Nephrol 7: 463-469, 1999.

69) Norman JT, Clark IM, Garcia PL. Hyopxia promotes fibrogenesis in human renal fibroblasts. Kidney Int 58: 2351-2366, 2000.

70) Zeisberg M, Strutz F, Muller GA. Role of fibroblast activation in inducing interstitial fibrosis. J Nephrol 13 (Suppl 3): S111-S120, 2000.

71) Manotham K, Tanaka T, Matsumoto M, et al. Transdifferentiation of cultured tubular cells induced by hypoxia. Kidney Int (in press).

72) Weinberg JM, Venkatachalam MA, Roeser NF, Nissim I. Mitochondrial dysfunction during hypoxia/reoxygenation and its correction by anaerobic metabolism of citric acid cycle intermediates. Proc Natl Acad Sci 97: 2826-2831, 2000.

73) Tanaka T, Hanafusa N, Ingelfinger JR, Ohse T, Fujita T, Nangaku M. Hypoxia induces apoptosis in SV40-immortalized rat proximal tubular cells through the mitochondrial pathways, devoid of HIF-1-mediated upregulation of Bax. Biochem Biophys Res Commun 309: 222-231, 2003.

74) Tanaka $T$, Miyata $T$, Inagi $R$, et al. Hypoxia-induced apoptosis in cultured glomerular endothelial cells-involvement of mitochondrial pathways. Kidney Int 64: 2020-2032, 2003.

75) Khan S, Cleveland RP, Koch CJ, Schelling JR. Hypoxia induces renal tubular epithelial cell apoptosis in chronic renal disease. Lab Invest 79: 1089-1099, 1999.

76) Macconi D, Ghilardi M, Bonassi ME, et al. Effect of angiotensinconverting enzyme inhibition on glomerular basement membrane permeability and distribution of zonula occludens-1 in MWF rats. J Am Soc Nephrol 11: 477-489, 2000.

77) Benigni A, Tomasoni S, Gagliardini E, et al. Blocking angiotensin II synthesis/activity preserves glomerular nephrin in rats with severe nephrosis. J Am Soc Nephrol 12: 941-948, 2001.

78) Ruggenenti P, Mosconi L, Vendramin G, et al. ACE inhibition improves glomerular size selectivity in patients with idiopathic membranous nephropathy and persistent nephrotic syndrome. Am J Kidney Dis 35: 381-391, 2000.

79) Remuzzi A, Perico N, Sangalli F, et al. ACE inhibition and ANG II receptor blockade improve glomerular size-selectivity in IgA nephropathy. Am J Physiol Renal Physiol 276: F457-F466, 1999.

80) Jafar TH, Schmid CH, Landa M, et al. Angiotensin-converting enzyme inhibitors and progression of nondiabetic renal disease. A metaanalysis of patient-level data. Ann Intern Med 135: 73-87, 2001.

81) Agodoa LY, Appel L, Bakris GL, et al, African American Study of Kidney Disease and Hypertension (AASK) Study Group. Effect of ramipril vs amlodipine on renal outcomes in hypertensive nephrosclerosis: a randomized controlled trial. JAMA 285: 2719-2728, 2001.

82) Jafar TH, Stark PC, Schmid CH, et al, AIPRD Study Group. Proteinuria as a modifiable risk factor for the progression of nondiabetic renal disease. Kidney Int 60: 1131-1140, 2001.

83) Nangaku M. Complement regulatory proteins-are they important in disease? J Am Soc Nephrol 14: 2411-2413, 2003.

84) Jungers P, Choukroun G, Oualim Z, Robino C, Nguyen AT, Man NK. Beneficial influence of recombinant human erythropoietin therapy on the rate of progression of chronic renal failure in predialysis patients. Nephrol Dial Transplant 16: 307-312, 2001.

85) Roth D, Smith RD, Schulman G, et al. Effects of recombinant human erythropoietin on renal function in chronic renal failure predialysis patients. Am J Kidney Dis 24: 777-784, 1994.

86) Kuriyama S, Tomonari H, Yoshida H, Hashimoto T, Kawaguchi $Y$, Sakai O. Reversal of anemia by erythropoietin therapy retards the progression of chronic renal failure, especially in nondiabetic patients. Nephron 77: 176-185, 1997.

87) Kang DH, Hughes J, Mazzali M, Schreiner GF, Johnson RJ. Impaired angiogenesis in the remnant kidney model: II. Vascular endothelial growth factor administration reduces renal fibrosis and stabilizes renal 


\section{Tubulointerstitial Injury and ESRD}

function. J Am Soc Nephrol 12: 1448-1457, 2001.

88) Kim YG, Suga SI, Kang DH, et al. Vascular endothelial growth factor accelerates renal recovery in experimental thrombotic microangiopathy. Kidney Int 58: 2390-2399, 2000.

89) Suga S, Kim YG, Joly A, et al. Vascular endothelial growth factor (VEGF121) protects rats from renal infarction in thrombotic microangiopathy. Kidney Int 60: 1297-1308, 2001.

90) Kunter U, Rong S, van Roeyen C, et al. Systemic overexpression of VEGF165 aggravates mesangioproliferative nephritis in rat. Nephrol Dial Transplant 18 (Suppl 4): 569, 2003 (Abstract).

91) Hofer T, Wenger R, Gassmann M. Oxygen sensing, HIF-1alpha stabilization and potential therapeutic strategies. Eur J Physiol 443: 503507, 2002.

92) Ivan M, Haberberger T, Gervasi DC, et al. Biochemical purification and pharmacological inhibition of a mammalian prolyl hydroxylase acting on hypoxia-inducible factor. Proc Natl Acad Sci 99: 13459-13464, 2002.

93) Warnecke C, Griethe W, Weidemann A, et al. Activation of the hypoxia-inducible factor-pathway and stimulation of angiogenesis by application of prolyl hydroxylase inhibitors. FASEB J 17: 1186-1188, 2003.

94) Willam C, Masson N, Tian YM, et al. Peptide blockade of HIFalpha degradation modulates cellular metabolism and angiogenesis. Proc Natl Acad Sci 99: 10423-10428, 2002.

95) Shyu KG, Wang MT, Wang BW, et al. Intramyocardial injection of naked DNA encoding HIF-1 alpha/VP16 hybrid to enhance angiogenesis in an acute myocardial infarction model in the rat. Cardiovasc Res 54: 576-583, 2002.

96) Vincent KA, Shyu KG, Luo Y, et al. Angiogenesis is induced in a rabbit model of hindlimb ischemia by naked DNA encoding an HIF-1 alpha/VP16 hybrid transcription factor. Circulation 102: 2255-2261, 2000.

97) Michaud SE, Menard C, Guy LG, Gennaro G, Rivard A. Inhibition of hypoxia-induced angiogenesis by cigarette smoke exposure: impairment of the HIF-1 alpha/VEGF pathway. FASEB J 17: 1150-1152, 2003.

98) Yuan Y, Hilliard G, Ferguson T, Millhorn DE. Cobalt inhibits the interaction between hypoxia-inducible factor-alpha and von Hippel-Lindau protein by direct binding to hypoxia-inducible factor-alpha. J Biol Chem 278: 15911-15916, 2003.

99) Matsumoto M, Makino Y, Tanaka T, et al. Induction of renoprotective gene expression by cobalt ameliorates ischemic injury of the kidney in rats. J Am Soc Nephrol 14: 1825-1832, 2003. 